\title{
A retrospective review of trampoline-related injuries presenting to a paediatric emergency department in Singapore
}

Francesca May Ting $\underline{\operatorname{Lim}}^{1}$, MBBS, Vigil $\underline{\text { James }}^{1}, \mathrm{MBBS}, \mathrm{MRCPCH}, K$ hai Pin $\underline{\operatorname{Le}}{ }^{1}$, MRCPCH, MMed, Sashikumar Ganapathy ${ }^{1}$, MB BCh

\begin{abstract}
INTRODUCTION Trampolining is a popular activity. However, to our knowledge, no studies on paediatric trampolinerelated injuries (TRIs) have been conducted in Asia. We aimed to provide an Asian perspective on paediatric TRIs and evaluate current safety measures.

METHODS Patients aged under 16 years who presented to the emergency department at KK Women's and Children's Hospital, Singapore, from March 2012 to June 2016 with a TRI were identified from the National Trauma Registry. Data was collated retrospectively focusing on age, location of the trampoline, mechanism and location of injury, treatment, disposition, and follow-up treatment.

RESULTS 137 children were seen for a TRI during this period. There was even representation across age groups (< 6 years, $6-11$ years and $11-16$ years). $60.6 \%$ of these injuries occurred in a public trampoline park, and a smaller proportion involved home and school trampolines. $61.3 \%$ of injuries occurred on the trampoline and $25.5 \%$ involved a fall off it, while the remaining were incurred by hitting the trampoline frame. The most common injury was soft tissue injury, followed by fractures and dislocations, of which $16.7 \%$ required surgical intervention. Most patients were discharged to an outpatient clinic. $14.6 \%$ of all patients required admission and $9.5 \%$ eventually required surgical intervention. There were three stable head injuries and no cervical spine injuries or deaths.

CONCLUSION The existence of trampoline parks has contributed to a rise in TRIs. We recommend measures such as general education, changes in the setup around the trampoline, increasing the age limit for trampolining, adult supervision and discouraging double bouncing.
\end{abstract}

Keywords: injuries, paediatric, Singapore, trampoline

\section{INTRODUCTION}

Trampolining is a popular activity today; what started off as a competitive sport has become a well-received recreational activity among children and adults. Within the last five years, Singapore has seen more than five recreational trampoline parks open throughout the island. Most of these trampoline parks consist of a number of interconnected trampolines, surrounded by protective nets along its periphery. Other features of the trampoline park include foam pits, a parkour wall and a slam dunk zone. These parks also hold beginners' trampoline classes for novice children. Trampoline parks have also become a popular location to hold events for children. ${ }^{(1-3)}$

Multiple studies have described an increase in the number of injuries among children as a result of trampoline-related activities. The National Electronic Injury Surveillance System sample described multiple cases of spinal cord injuries as well as a traumatic brain injury and two deaths at trampoline parks. ${ }^{(4-6)}$ Several other studies also pointed out trampoline-related injuries (TRIs) as a significant cause of cervical spine injury, which can subsequently cause long-term neurological sequelae. .5,7-11) $^{\text {(1) }}$

The American Academy of Pediatrics published a study in 1977 recommending a ban on trampoline activities as part of physical education programmes in schools and that trampolining should be abolished as a competitive sport, only to review and soften their stance in the 1980s. ${ }^{(12-14)}$ In response, the American Academy of Orthopaedic Surgeons recommended some safety measures that should be put in place when using the trampoline. ${ }^{(15)}$

This study aimed to provide an Asian perspective of paediatric TRIs by retrospectively reviewing the data of patients (aged 0-16 years) who presented to the emergency department (ED) in KK Women's and Children's Hospital (KKH), the largest children's hospital in Singapore, for a TRI between 2012 and 2016. We focused on the types and mechanisms of injury, treatment required, and the morbidities and mortalities of the injury. In doing so, we aimed to evaluate the effectiveness of the safety measures in place and recommend further measures that should be reinforced.

\section{METHODS}

A single-centre retrospective study was conducted on all children under the age of 16 years who had presented to the ED of $\mathrm{KKH}$ for a TRI between March 2012 and June 2016. Approval was granted by the SingHealth Centralised Institutional Review Board. All data was obtained from the National Trauma Registry and all subjects who met the inclusion criteria were selected from the registry, regardless of whether they were on the trampoline for recreational or competitive purposes. Patients who presented with any other pre-existing comorbid condition (in addition to the TRI) that might affect their eventual disposition were excluded. 
Data was collated focusing on these features: age group, whether the trampoline was part of a public trampoline park or a privately owned trampoline, the exact day and date of the injury, time of accident and presentation, cause of fall, mechanism of injury, part of body injured, type of injury, treatment in the ED, disposition, and follow-up treatment. All fractures were correlated with radiological findings.

Data was interpreted by a statistician using IBM SPSS Statistics version 23.0 (IBM Corp, Armonk, NY, USA). Descriptive data for categorical variables was presented as number of cases and percentages, while differences across groups were expressed using chi-square test or Fisher's exact test, as appropriate. A p-value $<0.05$ was considered statistically significant.

\section{RESULTS}

During the study period from March 2012 to June 2016, a total of 137 children presented to $\mathrm{KKH}$ for TRIs. Of these, 73 (53.3\%) were female and 64 (46.7\%) were male. 52 (38.0\%), 49 (35.8\%) and 36 (26.3\%) of the patients were in the $6-11$ years, $11-16$ years and $<6$ years age groups, respectively. $60.6 \%$ had injured themselves in a public trampoline park, $24.8 \%$ were injured through trampoline use in school and $14.6 \%$ had fallen while using a trampoline at home. The demographic profile of the patients is summarised in Table I.

Most children had injured themselves directly through a failed landing while jumping on the trampoline, with 84 (61.3\%) injuries being sustained on the trampoline plane. 35 (25.5\%) injuries were sustained outside the trampoline and 10 (7.3\%) by falling on the trampoline frame. Another $8(5.8 \%)$ falls were not adequately classified. 7 (5.1\%) children had accidentally collided against the trampoline frame while playing in the vicinity of the trampoline. In 10 (7.3\%) of the trampoline injuries, another person was present and jumping concurrently with the child; 5 (50.0\%) of these children lost balance and fell on or off the trampoline, while the other $5(50.0 \%)$ were hit or stepped on by another person while they were jumping together.

The anatomical areas that were most commonly involved in the trampoline accidents were arms and legs, with a total of $110(80.3 \%)$ injuries involving the limbs (Table II). Lower limb injuries were most common in the $<6$ years and $11-16$ years age groups. However, upper limb injuries were mostly in the 6-11 years and 11-16 years age groups (Fig. 1). Overall, three head injuries were recorded during the study period, of which two were in the $<6$ years group (Table III). Soft tissue injuries were the most common type of injuries sustained, comprising $59.1 \%$ $(n=81)$ of the 137 patients. These included contusions, abrasions, lacerations, ligamentous strains and joint sprains. The next most common type of injuries were fractures and dislocations, which occurred in $43.8 \%(n=60)$ patients. Four patients presented with two types of injuries involving both soft tissue injuries and fractures. The incidence of soft tissue injuries was highest among the older children ( $6-16$ years) when compared to the $<6$ years age group, and this was statistically significant $(p=0.02)$.

The incidence of fractures was highest in the 11-16 years age group, but this was not statistically significant $(p=0.26$ ). The number of fractures was evenly distributed among all three
Table I. Demographics of the patients $(n=137)$.

\begin{tabular}{|c|c|}
\hline Variable & No. (\%) \\
\hline \multicolumn{2}{|l|}{ Year of visit } \\
\hline March-December 2012 & $13(9.5)$ \\
\hline January-December 2013 & $17(12.4)$ \\
\hline January-December 2014 & $38(27.7)$ \\
\hline January-December 2015 & $49(35.8)$ \\
\hline January-June 2016 & $20(14.6)$ \\
\hline \multicolumn{2}{|l|}{ Gender } \\
\hline Male & $64(46.7)$ \\
\hline Female & $73(53.3)$ \\
\hline \multicolumn{2}{|l|}{ Location of fall* } \\
\hline On the trampoline plane & $84(61.3)$ \\
\hline Outside the trampoline & $35(25.5)$ \\
\hline On the trampoline frame & $10(7.3)$ \\
\hline \multicolumn{2}{|l|}{ Age group (yr) } \\
\hline$<6$ & $36(26.3)$ \\
\hline 6 to $<11$ & $52(38.0)$ \\
\hline 11 to $<16$ & $49(35.8)$ \\
\hline \multicolumn{2}{|c|}{ Location of injury according to age group } \\
\hline Public trampoline & $83(60.6)$ \\
\hline$<6 y r$ & 24 \\
\hline 6 to $<11 \mathrm{yr}$ & 34 \\
\hline 11 to $<16 y r$ & 25 \\
\hline Home/private estate & $20(14.6)$ \\
\hline$<6 y r$ & 11 \\
\hline 6 to $<11 \mathrm{yr}$ & 6 \\
\hline 11 to $<16 y r$ & 3 \\
\hline School & $34(24.8)$ \\
\hline$<6 y r$ & 4 \\
\hline 6 to $<11 \mathrm{yr}$ & 10 \\
\hline 11 to $<16 \mathrm{yr}$ & 20 \\
\hline
\end{tabular}

*Location was not available for 8 falls.

age groups (Table III), and there was no significant difference $(p=0.78)$ between the genders of patients who sustained fractures. Of the fractures sustained, 34 were in the upper extremity and 17 were in the lower extremity. One child presented with both an upper and lower limb fracture. Out of the 52 fractures, 18 (34.6\%) were around the elbow joint, including supracondylar and lateral condyle fractures, and fractures of the olecranon and radial neck. $9(17.3 \%)$ out of 52 patients who sustained a fracture underwent manual reduction of fracture in the ED. However, 10 (19.2\%) patients who sustained a fracture eventually had to undergo open reduction and internal fixation (ORIF) of the fracture. Overall, $25.0 \%, 35.3 \%$ and $47.4 \%$ of patients with fractures in the age groups of $<6$ years, $6-11$ years and $11-16$ years, respectively, required intervention for fracture stabilisation.

The majority of the patients $(75.9 \%, \mathrm{n}=104)$ were discharged from the ED with outpatient follow-up, mostly to the orthopaedic surgery department. 19 (13.9\%) patients did not require any form of follow-up, while $14(10.2 \%)$ required inpatient admission for specialist care. $6(4.4 \%)$ of the patients who were recommended for follow-up as outpatients were electively admitted for further 
treatment. Overall, 20 (14.6\%) patients required inpatient admission and $13(9.5 \%)$ patients eventually required surgical intervention.

\section{DISCUSSION}

Although the data only looked at ED visits over four years, there has been an apparent increase in the number of visits to the ED for TRIs, which may be related to the increase in prevalence of trampolines and trampoline parks in Singapore. Although most injuries were mild, close to $10 \%$ required surgical intervention.

The most common injuries sustained were soft tissue injuries followed by fractures, consistent with many other studies

Table II. Distribution of injuries $(n=137)$.

\begin{tabular}{|ll|}
\hline Variable & No. (\%) \\
\hline Injured body part & $7(5.1)$ \\
\hline Head & $9(6.6)$ \\
\hline Face and oral cavity & $1(0.7)$ \\
\hline Neck & $48(35.0)$ \\
\hline Arms & $2(1.5)$ \\
\hline Chest & $8(5.8)$ \\
\hline Back & $62(45.3)$ \\
\hline Legs & \\
\hline Type of injury* & $81(59.1)$ \\
\hline Soft tissue injuries & $60(43.8)$ \\
\hline Fractures/dislocations & $3(2.2)$ \\
\hline Head injuries & \\
\hline Disposition & $14(10.2)$ \\
\hline Admitted & $19(13.9)$ \\
\hline Discharged & $104(75.9)$ \\
\hline Discharged with outpatient follow-up & $6(4.4)$ \\
\hline Electively admitted at a later date &
\end{tabular}

*Some patients had more than 1 type of injury. conducted. ${ }^{(6,7,16,17)}$ Certain trends relating to age range and type of injury were also noted. While fractures appeared to be common across all three age groups, contusions and ligamentous strains were more common in those who were aged 6-16 years (Table III). A study in Norway showed that fractures were more common in children aged below ten years, who are presumably at greater risk because of immature judgement, coordination and strength, and anatomical characteristics such as open bone physes. ${ }^{(7)}$

A significant proportion of those with fractures in our population $(\mathrm{n}=19,36.5 \%)$ required intervention either via manipulation and reduction in the ED or surgical reduction, while the majority were conservatively managed and discharged with no further complications. The majority of patients who had fractures requiring manual or surgical reduction were aged 11 years and above. Notably, $47.4 \%$ of those in this age range with a fracture required intervention (Table III), suggesting a possible trend of more severe fractures occurring in the older age group. Although there is insufficient information on the exact mechanisms that caused each fracture, slightly more than half (53.8\%, seven out of 13 ) of the injuries that eventually required surgical intervention occurred during physical training for competitive trampolining. Among these, ten were for ORIF of fractures, while three were for ligament repair. It can be assumed that injuries that occur during physical training sessions are likely to be different from those sustained during recreational trampolining, and that the injury risk and severity of injury would be higher as the complexity of trampolining techniques increases. ${ }^{(18)}$ This also suggests that even if a rise in the number of public trampoline parks results in an increase in TRIs, it may not cause increased morbidity and mortality.

While cervical spine injuries are a common cause of neurological sequelae and morbidity in TRIs in other studies, ${ }^{(5,8-11)}$ only one neck injury, which was attributed to a muscular strain, presented to our ED. Of the three head injuries sustained in our population, two occurred in those below six years of age, and

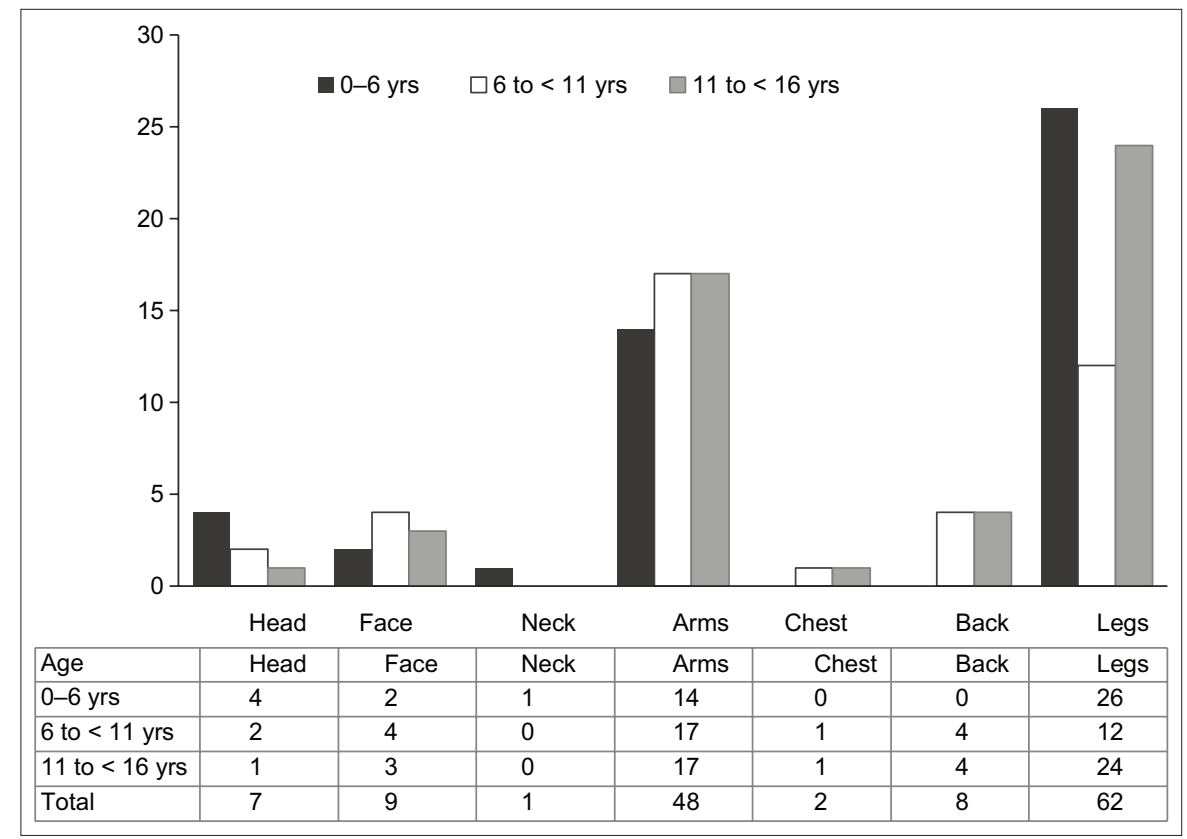

Fig. 1 Bar chart and table show the age range of the children and location of the injury. 
Table III. Types of injuries and their outcome according to the age of the patients.

\begin{tabular}{|c|c|c|c|c|}
\hline \multirow[t]{2}{*}{ Variable } & \multicolumn{3}{|c|}{ No. } & \multirow[t]{2}{*}{ p-value } \\
\hline & $<6 \mathrm{yr}$ & 6 to $<11 \mathrm{yr}$ & 11 to $<16 \mathrm{yr}$ & \\
\hline \multicolumn{5}{|l|}{ Type of injury by age } \\
\hline Soft tissue injuries & 15 & 33 & 33 & 0.02 \\
\hline Fractures/dislocations & 17 & 17 & 26 & 0.26 \\
\hline Head injuries & 2 & 1 & 0 & 0.36 \\
\hline \multicolumn{5}{|l|}{ Fracture description } \\
\hline No. of fractures & 16 & 17 & 19 & \\
\hline Gender & & & & 0.78 \\
\hline Male & 7 & 9 & 11 & \\
\hline Female & 9 & 8 & 8 & \\
\hline Required manual fracture reduction alone & 1 & 4 & 4 & \\
\hline Required surgical reduction & 3 & 2 & 5 & \\
\hline
\end{tabular}

all three when the child fell off the trampoline: one was due to double bouncing, while the other two were unwitnessed falls. Head injuries tend to occur when a child falls off the trampoline. ${ }^{(19)}$ All three patients with head injuries were admitted to the neurosurgical ward for monitoring, remained well and were discharged the following day. One of them had a concurrent uncomplicated laceration of the face requiring suturing.

This study has shown that the majority of the injuries were sustained when the child had a failed landing on the trampoline plane $(n=84)$ and relatively fewer injuries $(n=35)$ were a result of the child bouncing off the trampoline onto the ground outside the trampoline plane. Injuries that happened outside the trampoline plane tended to result in a head injury or a fall on an outstretched hand, resulting in an upper limb injury. While there were cases of children falling off the trampoline at public trampoline parks, most of these injuries occurred in a private setting - either in school, at home or in a residential estate. The data reviewed had insufficient information regarding the safety settings around the trampoline that each child was jumping on.

A significant proportion ( $\mathrm{n}=34,24.8 \%$ ) of injuries were sustained through the use of trampolines in schools. However, the greatest number of TRIs still occurred in public trampoline parks ( $n=83,60.6 \%)$, suggesting that the increase in popularity and accessibility to public trampoline parks in Singapore may indeed contribute to an increase in TRIs. Out of 20 admissions, only $2(10 \%)$ had injuries sustained on a home trampoline.

A study conducted by Linakis et al on ED visits in the United States for paediatric TRIs concluded that the relatively inexpensive home trampoline has made home trampoline use very popular, and its use continues to be a significant source of childhood injury morbidity. ${ }^{(16)}$ There are other studies conducted overseas in which the majority of TRIs were sustained on home trampolines. ${ }^{(4,17)}$ However, this is not the case in Singapore, probably due to space constraints and the number of trampoline parks within a short distance of each other. Travelling to a public trampoline park may be much more convenient in Singapore.

Several studies have correspondingly identified that double bouncing, or having multiple users on a trampoline, was a significant cause of injury. ${ }^{(6,16,18,20-22)}$ In this study, ten injuries were caused by multiple users being on the trampoline, resulting in the injured child either falling off the trampoline due to a heavier force on the trampoline or being involved in a collision. Consistent with the aforementioned studies, the injuries in which multiple users were on the trampoline happened more often in younger children, with six out of ten of them occurring in children aged below six years and none in the 11-16 years group. All the injuries from double bouncing were minor and did not require any long-term follow-up.

Several studies have evaluated the effectiveness of safety measures in trampoline use, and most reported that safety measures have minimal impact on the frequency of TRIs. ${ }^{(7,19,23-26)}$ Ashby et al showed that a voluntary standard in Australia had little effect on spring and frame injuries, and none on multipleuser injuries. ${ }^{(24)}$ Nysted and Drogset concluded that trampoline nets may not have helped to reduce TRIs at all. ${ }^{(7)}$

In Singapore, safety rules and features have been put in place in public trampoline parks. The safety nets surrounding the periphery of public trampolines may have served their purpose, as only a minority of falls off trampolines occurred in public trampoline parks. Another rule is that children aged below 18 years require a parent or guardian's approval, and all children below four years need to be supervised by an adult. Additionally, all users are required to wear grip socks to prevent slipping, and all pockets are to be emptied, with no handheld objects allowed during jumping. Of note, none of the children who visited our ED for TRIs had sustained injuries from handheld objects. Flipping is allowed but at one's own risk. (1) Studies and policies overseas also suggest that adult or parental supervision would significantly help to reduce TRIs. ${ }^{(4,12-14,22)}$ However, there was no documentation on the presence of supervision in the data to sufficiently conclude if adult supervision had been adequate during trampoline use.

While most trampolines sold for home use have standard safety measures such as padded frames, these have shown to become ineffective over time. Moreover, private users may choose not to use additional safety equipment that may be sold with the trampoline. Ten of the injuries in our study were a result of the child falling directly onto the frame. Two children sustained lacerations to the face or head that required toileting 
and suturing, one required an ORIF and one sustained a fracture that was manually reduced in the ED. However, in three of these injuries, the child had been engaged in an activity that was not on the trampoline and had accidentally hit the frame, while another was a result of a child not following safety measures. It may be useful for outlets selling trampolines to ensure that buyers are adequately educated on the risks associated with trampoline use by providing safety handouts for users and caretakers. It should also be mandatory to have soft mats placed around the periphery of the trampoline. Double bouncing should be discouraged on home trampolines. Schools and homes can also take measures to install trampolines away from spaces where children are engaging in other sports activities.

This study was not without limitations. Being a retrospective study, the data collected depended entirely on the information documented by the ED physician who attended to the child on the day of presentation. As such, the amount of information collected for each ED visit varied. There was some incomplete data regarding the mechanism of injury, the location of injury, the design of the trampoline and the safety measures in place, limiting our assessment of the adequacy of these safety measures. Furthermore, this study also only examined a representative sample of ED visits. There was no way to ascertain the number of TRIs that did not present to our hospital, hence our findings may not be representative of the total number of paediatric TRIs in Singapore. As the aim of this study was to focus on the epidemiology and characteristics of each TRI, each child was not followed up on for long-term complications, such as neuropathies, or the psychological impact of the fall, although we noted that some children required long-term rehabilitation.

Further studies can be conducted in future with a larger sample size, to explore other factors such as the safety measures in place, type of trampoline used, size of the trampoline, body mass index of the injured patient, the presence of other users on the same trampoline, extent of parental supervision, and whether the child was performing a stunt. In addition, they can examine whether experience can reduce the number and severity of TRIs, such as comparing the number of days of trampoline experience and correlating it with the severity of the injury.

In conclusion, this study has found that the increase in the prevalence of trampolines has resulted in an increase in ED visits for TRIs, although the majority of the TRIs seen at our hospital were mild, with no deaths or severe neurological sequelae. To date, there have been many studies supporting regulation or banning of trampoline activities. However, trampolining is an engaging indoor activity that helps to improve motor control in children, and there are risks of falls and injuries as with all forms of physical activity. Instead of a ban, we would recommend that additional safety measures be taken, such as adequate education when selling trampolines, ensuring adult supervision, mandatory placement of mats around the periphery of all trampolines and restricting trampoline use to one person. Public trampoline parks can also consider increasing the age limit for public trampoline use.

\section{ACKNOWLEDGEMENTS}

We sincerely thank Ms Ng Wai Yee, Statistician, Department of Biostatistics, National University of Singapore, Singapore, for assisting in the interpretation of data. We also sincerely thank all of the ED doctors in $\mathrm{KKH}$ for contributing to the data collected as well as the staff of the National Trauma Registry, from whom we obtained the data.

\section{REFERENCES}

1. AMPED Trampoline Park Singapore. Available at: https://ampedsingapore.com/. Accessed June 17, 2017.

2. Katapult Trampoline Park. Available at: http://katapult.com.sg/. Accessed June 17, 2017.

3. Zoom Park Singapore. Available at: http://zoomparkasia.sg/. Accessed June 17, 2017

4. Council on Sports Medicine and Fitness, American Academy of Pediatrics, Briskin S, LaBotz M. Trampoline safety in childhood and adolescence. Pediatrics 2012; 130:774-9.

5. Furnival RA, Street KA, Schunk JE. Too many pediatric trampoline injuries. Pediatrics 1999; 103:e57.

6. Mulligan CS, Adams S, Brown J. Paediatric injury from indoor trampoline centres. Inj Prev 2017; 23:352-4.

7. Nysted M, Drogset JO. Trampoline injuries. Br J Sports Med 2006; 40:984-7.

8. Brown PG, Lee M. Trampoline injuries of the cervical spine. Pediatr Neurosurg 2000; 32:170-5.

9. Silver JR, Silver DD, Godfrey JJ. Trampolining injuries of the spine. Injury 1986; $17: 117-24$

10. Larson BJ, Davis JW. Trampoline-related injuries. J Bone Joint Surg Am 1995; 77:1174-8

11. Leonard H, Joffe AR. Children presenting to a Canadian hospital with trampolinerelated cervical spine injuries. Paediatr Child Health 2009; 14:84-8.

12. Committee on Accident and Poison Prevention, American Academy of Pediatrics. Policy statement: trampolines. Evanston, IL: American Academy of Pediatrics; 1977

13. Holroyd HJ, Garrettson LK, Greensher J, et al. Trampolines II. Pediatrics 1981; 67:438.

14. Trampolines at home, school, and recreational centers. American Academy of Pediatrics. Committee on Injury and Poison Prevention and Committee on Sports Medicine and Fitness. Pediatrics 1999; 103(5 Pt 1):1053-6.

15. American Academy of Orthopaedic Surgeons. Trampolines and trampoline safety. Position statement. Available at: https://www.aaos.org/uploadedFiles/ PreProduction/About/Opinion_Statements/position/1135\%20-\%20 Trampolines\%20and\%20Trampoline\%20Safety.pdf. Accessed July 20, 2017.

16. Linakis JG, Mello MJ, Machan J, Amanullah S, Palmisciano LM. Emergency department visits for pediatric trampoline-related injuries: an update. Acad Emerg Med 2007; 14:539-44.

17. Smith GA. Injuries to children in the United States related to trampolines, 19901995: a national epidemic. Pediatrics 1998; 101(3 Pt 1):406-12.

18. Esposito PW, Esposito LM. The reemergence of the trampoline as a recreational activity and competitive sport. Curr Sports Med Rep 2009; 8:273-7.

19. Shankar A, Williams K, Ryan M. Trampoline-related injury in children. Pediatr Emerg Care 2006; 22:644-6.

20. Bhangal KK, Neen D, Dodds R. Incidence of trampoline related pediatric fractures in a large district general hospital in the United Kingdom: lessons to be learnt. Inj Prev 2006; 12:133-4.

21. Hurson C, Browne K, Callender O, et al. Pediatric trampoline injuries. J Pediatr Orthop 2007; 27:729-32.

22. Klimek PM, Juen D, Stranzinger E, Wolf R, Slongo T. Trampoline related injuries in children: risk factors and radiographic findings. World J Pediatr 2013; 9:169-74.

23. Ashby K, Cassell E. Safe trampolining-a persistent challenge. Injury Prevention. 2012;18(Suppl 1):A87-A.

24. Ashby K, Eager D, D'Elia A, Day L. Influence of voluntary standards and design modifications on trampoline injury in Victoria, Australia. Inj Prev 2015; 21:314-9.

25. Morrongiello BA, Major K. Influence of safety gear on parental perceptions of injury risk and tolerance for children's risk taking. Inj Prev 2002; 8:27-31.

26. Alexander K, Eager D, Scarrott C, Sushinsky G. Effectiveness of pads and enclosures as safety interventions on consumer trampolines. Inj Prev 2010; 16:185-9. 